\title{
Review on progressive microforming of bulk metal parts directly using sheet metals (Keynote Paper)
}

\author{
M.W. Fu ${ }^{\mathrm{a}}$ and B. Meng \\ Department of Mechanical Engineering, The Hong Kong Polytechnic University, Hung Hom, \\ Kowloon, Hong Kong
}

\begin{abstract}
Due to the ubiquitous trend of product miniaturization, energy saving and weight reduction, micro/meso-scale parts have been widely used in many industrial clusters. Micromanufacturing processes for production of such micro/meso-scale parts are thus critically needed. Microforming, as one of these micro manufacturing processes, is a promising process and thus got many explorations and researches. Compared with the research on size effect affected deformation behaviours, less attention has been paid to the process development for mass production of micro-parts. The product quality and fabrication productivity of micro-parts depend on the involved process chain. To address the difficulty in handling and transporting of the micro-sized workpiece, development of a progressive microforming process for directly fabricating bulk micro-parts using sheet metals seems quite promising as it avoids or facilitates billet handling, transportation, positioning, and ejection in the process chain. In this paper, an intensive review on the latest development of progressive microforming technologies is presented. First of all, the paper summarizes the characteristic of progressive microforming directly using sheet metal. The size effect-affected deformation behaviour and the dimensional accuracy, deformation load, ductile fracture, and the surface finish of the microformed parts by progressive microforming using sheet metals are then presented. Finally, some research issues from the implementation of mass production perspective are also discussed.
\end{abstract}

\section{Introduction}

Micro/meso-scaled parts have been widely used in many industries including electronics, automobiles, jewellery, aerospace and biomedicine, etc. in tandem with the ubiquitous trend of product miniaturization $[1,2]$. However, the microforming process for mass production of miniaturized parts and its process chain have not yet been extensively studied and developed. From billet preparation perspective, there are two alternative approaches to substitute micromachining process, which is not so efficient. One is to prepare micro-billets via cutting metal wire, and the other is to directly blank bulk microparts from sheet metal. For the first one, it is difficult to control the quality of fracture surfaces of the preform. When the preform with the inclined fracture surfaces is used in the subsequent deformation operation, the deformation load in micro-punch may be unevenly distributed, resulting

\footnotetext{
${ }^{\text {a }}$ Corresponding author: mmmwfu@polyu.edu.hk
}

This is an Open Access article distributed under the terms of the Creative Commons Attribution License 4.0, which permits unrestricted use, distribution, and reproduction in any medium, provided the original work is properly cited. 
(a)

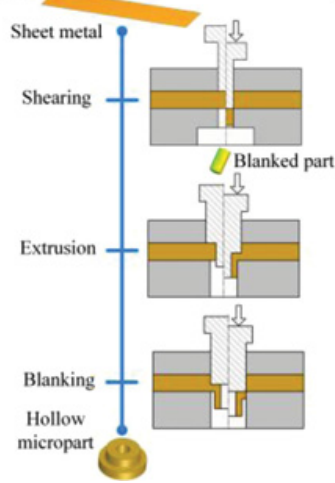

(b)

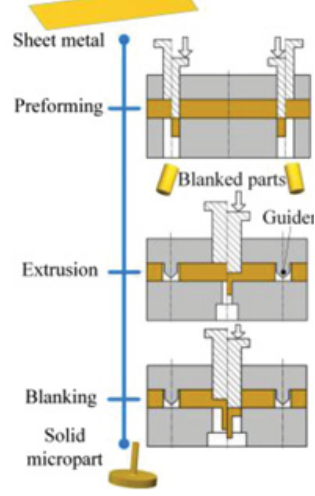

Figure 1. Flow chart of process chain for the hollow and solid micro-parts [10].

in the early failure of tooling. Whereas the second approach can address the common issues such as the adhesive forces induced by surface tension, van der Waals-force, and the electrostatic interaction between workpiece and handling tools in microforming process. To explore this process, Hirota [3] extruded micro-billet via pressing the sheet material in thickness direction. Fu and Chan $[4,5]$ further examined the feasibility of this process to fabricate the meso- and micro-scaled parts by directly using sheet metal, and identified its characteristics via scaling down the part size and using the materials with different grain sizes. Ghassemali et al. [6-9] further developed this process to manufacture the micro-pins by directly using copper strip, which consists of two simple steps, including extrusion and blanking. To ensure the precise positioning, the guide holes are performed on the edge of sheet metal, and the guide pins are utilized throughout the subsequent forming stages.

In this paper, the latest development of progressive microforming processes and the size effectaffected deformation behaviour are comprehensively reviewed. A panorama in terms of process realization and product quality control is provided to facilitate the design and development of microparts by using this unique microforming process, and further to advance this promising microforming process for mass production of micro/meso parts for different industrial clusters.

\section{Characteristic of progressive microforming}

In general, the progressively formed microparts can be classified into two categories: hollow and solid microparts. In forming of the hollow micro-parts, the pre-pierced hole in the sheet metal with the same dimension as the final part is first punched in the center of sheet metal, which is then used for positioning in the following operations. In fabrication of solid bulk micro-parts, the initial shearing is also conducted to pierce the guiding holes for accurate positioning in the sequential stages, as illustrated in Fig. 1 [10]. It can be seen that the characteristic of micro-shearing process customized for progressive microforming is to produce micro-scaled holes by using the sheet metals with different thicknesses. Figure 2 schematically illustrates the characteristics of the progressive microforming process developed by Ghassemali et al. [6-9] for the fabrication of a solid micro-part with a pin at its center. From the figure, it can be seen that the process set-up consists of two stages including pin forming by forward extrusion and blanking. In the first step, the metal strip is deformed by a punch with a defined diameter and a specified displacement. As a result, a portion of the material is forward extruded into the die cavity. The used copper strip is inserted via guides on the set-up. To ensure the precise positioning, guide holes are created on the edge of the strip and guide pins are used along the different stages in the process. 


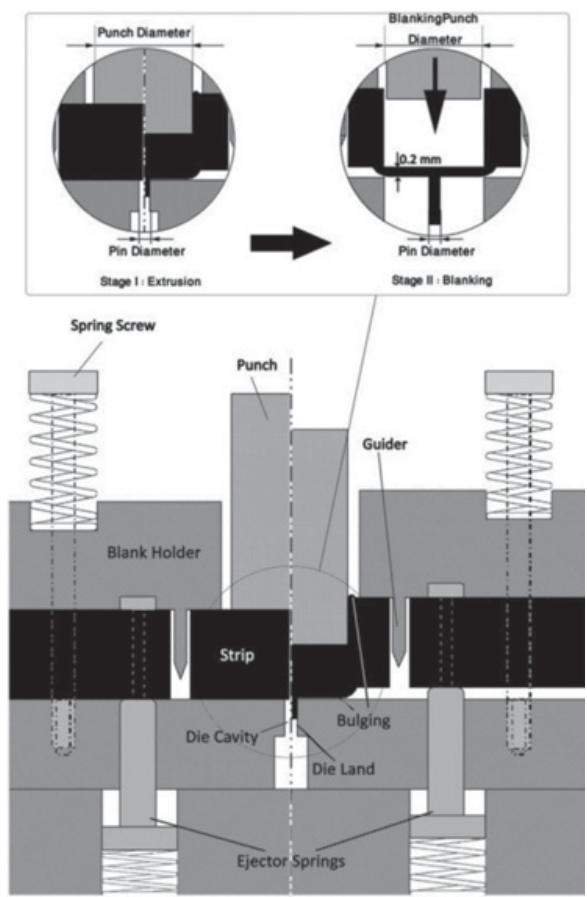

Figure 2. Schematic of the progressive microforming process for solid micro-parts [8].
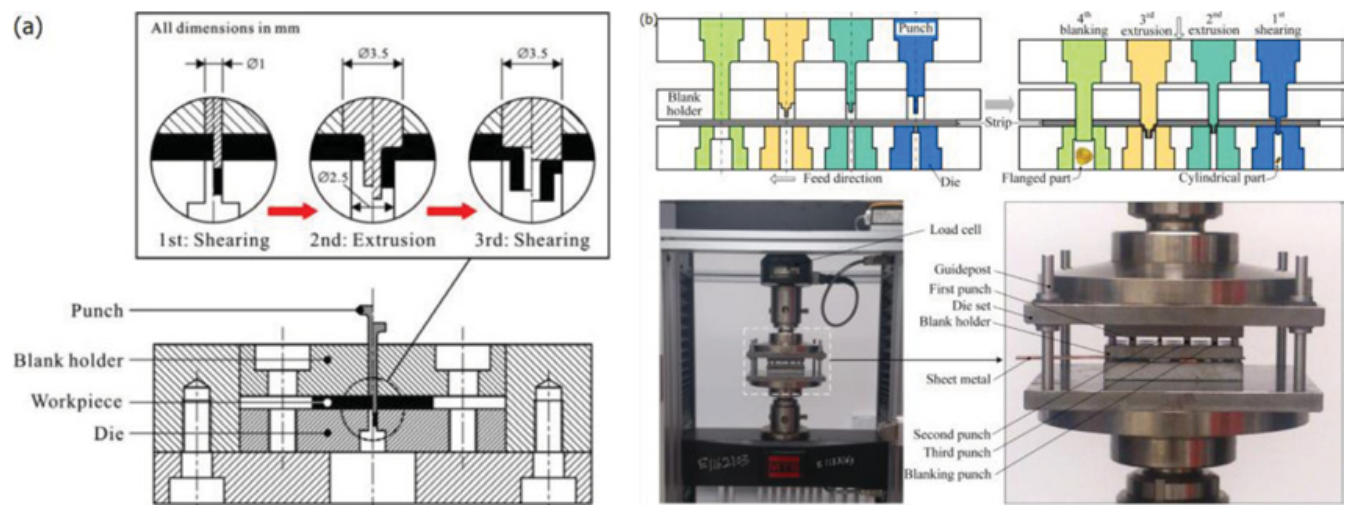

Figure 3. Progressive microforming system for fabrication of (a) micro-scale flanged parts $[4,5]$ and (b) two-level flanged part $[10,11]$.

The blank holder is spring-loaded with six springs. Then the sheet metal with the attached extruded pin is ejected by springs and can be transformed to the next stage [7].

Figure 3 shows the progressive microforming system developed by the authors' group to fabricate multi-step flanged parts. The formed meso- and microparts are presented in Fig. 4. During the forming process, an intermediate preform is fabricated with the sheet metal and the pre-pierced hole is used for positioning. The final part is blanked out from the sheet metal in the last operation. In such a way, the transporting, positioning, and ejection of the flanged part are facilitated. There are different processing operations in each stroke. In the first step, the sheet metal undergoes shearing deformation. 

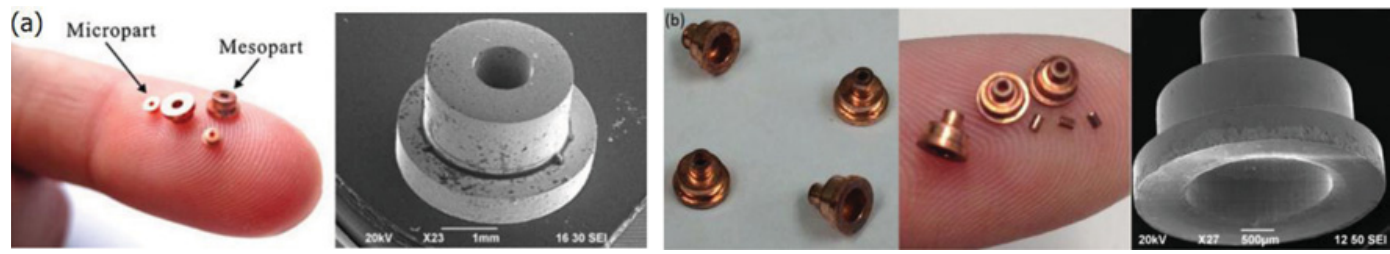

Figure 4. Progressively formed micro-part: (a) micro-scale flanged parts $[4,5]$ and (b) two-level flanged part $[10,11]$.

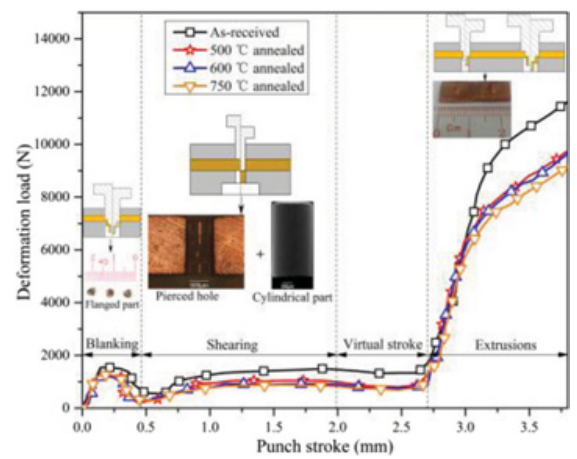

Figure 5. Load-stroke curve in progressive microforming [11].

A cylindrical part is blanked out and a hole in the sheet metal is pierced, which is used for positioning in the subsequent operations. In the second or third operation, the workpiece is extruded in thickness direction to form the multilevel flanged features, and a portion of the material is further pressed into the die orifice. It is noted that the formed part at the first three stages is attached to the sheet metal, which makes it easier for handling, positioning and transferring to the next step. In the last operation, the flanged part is removed from the strip by blanking operation. Considering the machine capacity and the mechanical characteristics of each forming operation, a different working procedure is arranged in one stroke via adjusting the punch length of each operation. The punch for blanking of the flanged part first contacts the sheet metal. After that, the piercing punch shears the cylindrical part and pierces a hole on the strip. Subsequently, the two extrusion processes with the same punch length are conducted simultaneously to form the multilevel flanged part [11].

\section{Deformation behaviour and ductile fracture}

The unique deformation behaviour and damage accumulation effect in the progressive microforming are crucial to process performance and the final product quality. Therefore, the key issues in terms of dimensional accuracy, material flow behaviour, fracture surface, and microstructure evolution of the formed parts during the progressive deformation should be well explored and addressed.

\subsection{Deformation behaviour}

In the progressive microforming process developed by $\mathrm{Fu}$ et al., the deformation load comes from different operations including shearing, extrusion and blanking. Figure 5 shows the load-stroke curves in the progressive microforming process under different conditions. It can be found that the deformation load of the as-received sheet metal is larger than that of the annealed ones. This is attributed to the fact 

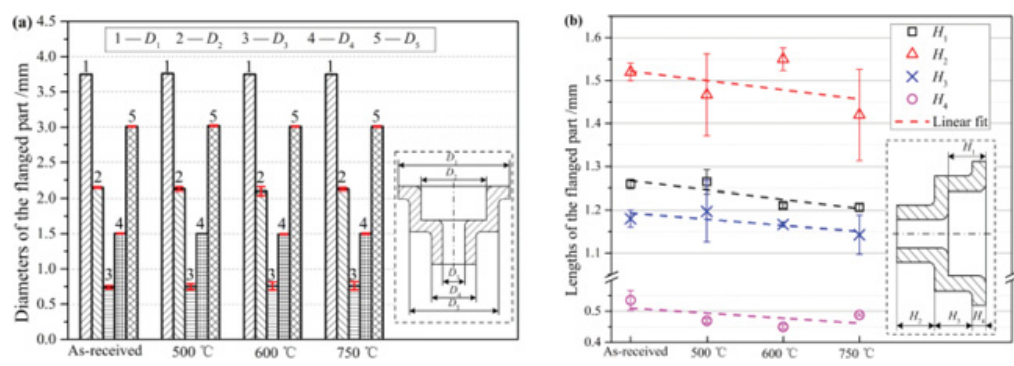

Figure 6. Dimensional variation of the flanged part: (a) diameter and (b) length [11].

that the as-received material was hardened in rolling process, in addition to grain boundary strengthening effect. However, the number of grains is reduced over the material thickness with annealing temperature, resulting in an inhomogeneous deformation and a largely localized deformation of the coarse-grained material $[10,11]$.

\subsection{Dimensional accuracy}

Dimensional accuracy is an important factor in micro-part development since it directly affects product quality and performance. In progressive microforming, the dimensional accuracy is mainly affected by material property, microstructure and process variables. Compared to macro-scale forming, there are new factors that increase the complexity of the process such as accumulative straining and inhomogeneous deformation. They are characterized by some common or specific phenomena that affect the dimensional accuracy of the fabricated parts. Figure 6 presents the dimensional variation of the two-level flanged parts. Compared with the fine-grained material, the standard deviation of geometric dimension with the coarse-grained material is enhanced, indicating the shape stability of the formed part worsens with the increase of grain size due to the inhomogeneous deformation. To fabricate the micro-parts for a given material, the desirable length of the extrudate can be obtained accurately via compensating the punch stroke and using the fine-grained material.

\subsection{Fracture surface}

The blanked surfaces of the materials with different states in the blanking process, the third operation of the progressive forming developed by Chan and Fu [4, 5], are shown in Fig. 7. The fracture mechanisms of the initiation, growth, and coalescence of micro-voids result in the rough surface finish. In addition, die design also affects the fracture surface in this process. The parabolic-shaped micro-voids occur on the fracture surfaces and the number of micro-voids decreases with the increase of grain size, as shown in Fig. 7. Furthermore, the size of fracture surface does not have a significant change with the decreasing number of grains over the thickness of workpiece.

Figure 8 shows the cross-section photographs of the two-level flanged part. It is observed that the extruded surface in the second operation is fractured with the corrugated surface, which is caused by void growth. The fracture is weakened with the increase of grain size, and there is no obvious ductile fracture for the material annealed at $750{ }^{\circ} \mathrm{C}$. This is because there is a small number of grains involved in extrusion deformation, meanwhile, the deformation degree and strain accumulation decline when the coarse-grained material is used. Another fracture pattern is micro-crack located on the sidewall of the flange, as shown in Fig. 9. The micro-crack results from the interactive effects of the second extrusion and blanking operation. Moreover, the micro-crack phenomenon is only observed in the asreceived material. The material is subjected to the severe strain accumulation in the second extrusion, 


\section{MATEC Web of Conferences}
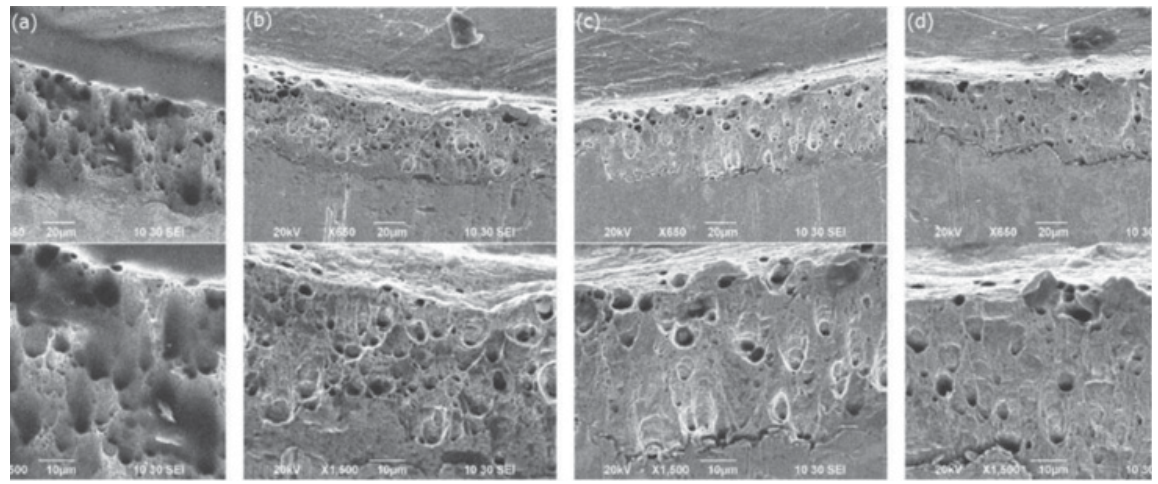

Figure 7. Fracture surfaces of the parts formed by micro progressive forming [5]. (a) As-received, (b) $500{ }^{\circ} \mathrm{C}$ annealed, (c) $600{ }^{\circ} \mathrm{C}$ annealed and (d) $750{ }^{\circ} \mathrm{C}$ annealed.

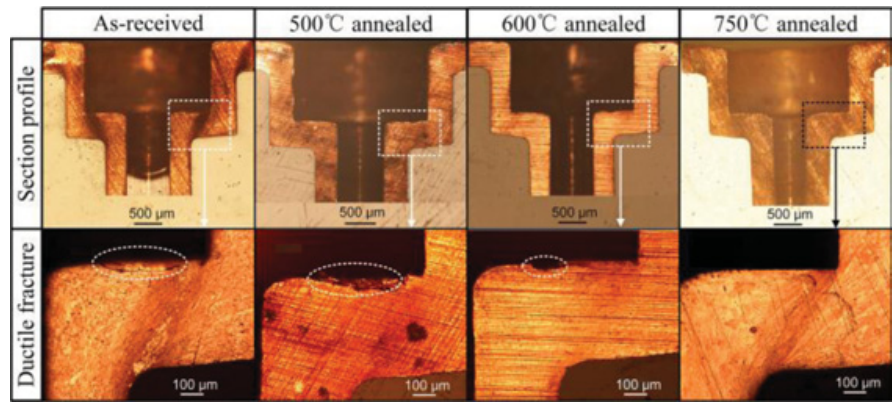

Figure 8. Fracture surfaces on the flanged part after the second micro-extrusion [11].
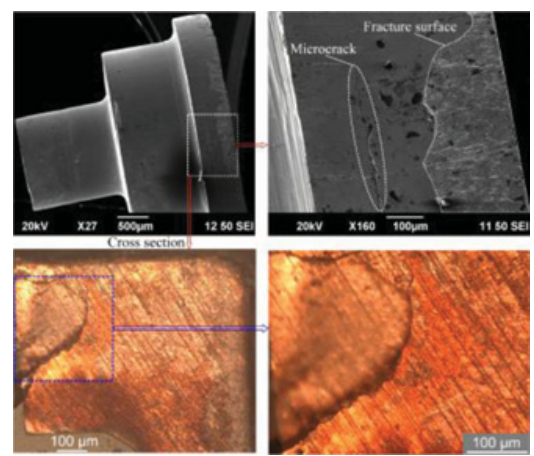

Figure 9. Micro-crack on the flanged part induced by the accumulative effect [11].

and the strain-induced fracture is generated on the extruded surface. Subsequently, the second flanged feature was blanked out from the sheet metal, and the fractured surface at the extrusion stage underwent extrusion and shearing deformation again. When the fractured surface was cut off from the copper sheet, the micro-crack occurred on the flange of the final workpiece. Figure 9 shows the internal characteristic of the micro-crack. It is found that the micro-crack extended into the flange about $200 \mu \mathrm{m}$ from the part edge, indicating the crack was formed before the blanking stage. 


\section{ICNFT 2015}

\section{Conclusions}

In this paper, the state-of-the-art researches and the latest development of progressive microforming processes are reviewed comprehensively. To overcome the difficulties in billet preparation and transportation and positioning of billet and preform in microforming processes, a unique microforming process directly using sheet metal to produce bulk micro-parts has been proposed. The progressive microforming process provides a possible solution to overcome these challenges. However, predicting the amount of extrusion sub-process and accumulative fracture phenomenon in progressive deformation is difficult. This is also critical to control the dimensional accuracy of the microformed parts using different materials.

The authors would like to acknowledge the funding support to this research from the General Research Fund of Hong Kong Government under the Project of 515012 (B-Q33F) and the projects of G-YBDM, G-YM93, G-U923 and G-UB59 from The Hong Kong Polytechnic University.

\section{References}

[1] T. Masuzawa, CIRP Ann. Manuf. Technol. 49, 473 (2000)

[2] M.W. Fu, W.L. Chan, Int. J. Adv. Manuf. Technol. 67, 2411 (2013)

[3] K. Hirota, J. Mater. Process. Technol. 191, 283 (2007)

[4] W.L. Chan, M.W. Fu, Mater. Des. 43, 249 (2013)

[5] M.W. Fu, W.L. Chan, Mater. Des. 49, 774 (2013)

[6] E. Ghassemali, A.E.W. Jarfors, M.J. Tan, S.C.V. Lim, Int. J. Mater. Form 6, 65 (2013)

[7] E. Ghassemali, M.J. Tan, A.E.W. Jarfors, S.C.V. Lim, Int. J. Adv. Manuf. Technol. 66, 611 (2013)

[8] E. Ghassemali, M.J. Tana, C.B. Wah, A.E.W. Jarfors, S.C.V. Lim, Mater. Sci. Eng. A 582, 379 (2013)

[9] E. Ghassemali, M.J. Tan, A.E.W. Jarfors, S.C.V. Lim, Int. J. Mech. Sci. 71, 58 (2013)

[10] B. Meng, M.W. Fu, C.M. Fu, J.L. Wang, Int. J. Mech. Sci. 93, 191 (2015)

[11] B. Meng, M.W. Fu, C.M. Fu, K.S. Chen, Mater. Des. (under review) 